Wojciech J. Piotrowski', Adam J. Białas' ${ }^{1}$, tukasz Gwadera', Anna Kumor-Kisielewska', Marek Fijałkowski², Zofia Kurmanowska', Jerzy Marczak', Witold Górski', Wojciech Angowski', Paweł Górski', Sylwia Kwiatkowska ${ }^{3}$

'Department of Pneumology and Allergy, Chair of Internal Medicine, Medical University of Lodz, Poland

${ }^{2}$ TB Unit, Regional Hospital for Tuberculosis and Lung Diseases, Tuszyn, Poland ${ }^{3}$ Department of Pneumology and Allergy,

Chair of Clinical Nursery, Medical University of Lodz, Poland

\title{
QuantiFERON-TB-GOLD In-Tube in patients with sarcoidosis
}

\begin{abstract}
Introduction: Sarcoidosis and tuberculosis (TB) are the diseases that share many similarities. Mycobacterium tuberculosis (MTB) culture results are the gold standard for the diagnosis of $\mathrm{TB}$, but false positive results are not rare.

The aim was to evaluate the utility of QFT in detecting latent TB infection in a group of sarcoidosis patients with negative history of TB and negative culture/BACTEC results, and checking sarcoidosis activity influence on the OFT results. Additionally, we assessed if QFT negative result may strengthen the suspicion that positive culture/BACTEC results are false positive.

Material and methods: 37 culture-negative and 6 culture-positive sarcoidosis patients were enrolled. On the basis of clinical and radiological data TB was considered unlikely (false-positive results). A control group consisted of age-matched subjects with excluded TB $(n=37)$. QuantiFERON-TB GOLD In-Tube (OIAGEN, USA) was used according to the manual. Test validity was checked basing on the results obtained from a low-risk $(n=21)$ and active TB group $(n=23)$.

Results: The frequency of positive results tended to be higher in MTB(-) sarcoidosis $(24.3 \%$ vs. $13.5 \%$ for the control group, $p=0.37$ ), but was similar to the general population. None of culture-positive sarcoidosis patients was QFT-positive. The positive results were equally distributed among patients with active and inactive sarcoidosis.

Conclusions: QFT has been found to be the useful test for the detection of latent TB infection in sarcoidosis patients. In addition, we confirm that sarcoidosis activity does not negatively influence the result of QFT. Moreover, OFT would be proposed as a cost-saving diagnostic test providing additional diagnostic information when false positive MTB culture result in the sarcoidosis patient is highly suspected. However, in each case clinical, radiological and epidemiological data should be considered before taking the therapeutic decision.
\end{abstract}

Key words: IGRA, quantiferon test, sarcoidosis, tuberculosis

Adv Respir Med. 2018; 86: 234-239

\section{Introduction}

Sarcoidosis and tuberculosis (TB) are the diseases that share many similarities responsible for difficulties in differential diagnosis. Mycobacterium tuberculosis (MTB) antigens have been suspected to induce sarcoidosis [1], and the coexistence of both entities seems to be possible [2]. The recent molecular studies have also revealed that the diseases have similar blood transcriptional signature [3, 4]. That is why, intriguing hypothesis of sarcoidosis and tuberculosis as the two ends of the same disease spectrum has been proposed [5].
Clear-cut differential diagnosis may be critical in a clinical setting, as misdiagnosis or oversight of TB potentially brings serious epidemiological threat. The gold standard for TB recognition is the positive culture result for MTB, however, negative sputum results do not exclude the disease. Moreover, false positive results are not infrequent, and have been reported even in reference laboratories [6].

The tuberculin skin test (TST) - negative in almost all sarcoidosis patients due to the peripheral anergy - is not a valuable tool for detecting latent TB infection in these patients, however, positive results in active sarcoidosis strongly

Address for correspondence: Wojciech J. Piotrowski, Department of Pneumology and Allergy, Chair of Internal Medicine, Medical University of Lodz, Kopcińskiego 22,

90-153 tódź, Poland, Tel. +48 4267875 05, e-mail: wojciech.piotrowski@umed.lodz.pl

DOI: 10.5603/ARM.2018.0037

Received: 24.07.2018

Copyright (C) 2018 PTChP

ISSN 2451-4934 
suggest coexistent TB [7]. In vitro IFN-gamma release assays (IGRAs), which have been developed in the last decade, are considered most accurate for detection of latent TB infection (LTBI) [8]. An alternative to TST, but with higher specificity and sensitivity, these tests identify individuals infected with MTB, and are of great importance in BCG-vaccinated population, like the Polish one. Upon the stimulation with mixture of antigens: early secretory antigenic target 6 (ESAT-6), culture filtrate protein 10 (CFP-10) and TB7.7, QuantiFERON-TB Gold In-Tube test measures IFN-gamma concentration by the use of ELISA. It is of note however, that this commercial test does not discriminate between patients with LTBI and those with active disease $[9,10]$.

The results of already published studies into the use of IGRA tests in the diagnosis of latent TB infection in sarcoidosis patients are not fully conclusive [11-13]. Although the authors are concordant as to the fact that positive results are not more frequent than in non-sarcoidosis control subjects, none of these studies provided data on sarcoidosis patients with suspected coexistent tuberculosis on the basis of positive culture results. Besides, data on the influence of the activity of sarcoidosis and consequent peripheral depletion of $\mathrm{T}$ cells on the final result of IGRA test are also scarce.

Therefore, questions to be addressed in the present study are the following: 1) What is the utility of QFT in detecting latent TB infection in a group of sarcoidosis patients with negative history of TB and negative culture/BACTEC results? 2) Does sarcoidosis activity influence the QFT results? 3) Can QFT negative result strengthen the suspicion that positive culture/BACTEC results are false positive?

\section{Material and methods}

\section{Study groups}

\section{Sarcoidosis - The MTB true negative}

Consecutive 37 patients admitted to the Department for the confirmation of sarcoidosis, and in whom the diagnosis was confirmed according to international guidelines [14] were included. In all patients, diagnostic bronchoscopy did not reveal MTB, neither in smear nor in culture examination. The previous treatment of TB or contact with a person known to have active infection were the exclusion criteria. This was assessed by a dedicated self-prepared questionnaire, and negative answers were required to all the questions below: Have you ever been told by a doctor to have TB? Have you ever been treated for TB? Have you ever been suspected of TB? Have you ever had any contacts with someone known to suffer from TB? This subgroup will be further indicated as $\mathrm{SarC}_{\mathrm{MTB}(-)}$ group. See table 1 for details of demographic and clinical data.

\section{Sarcoidosis - The MTB false positive}

An additional group consisted of six patients with the primary diagnosis of sarcoidosis (criteria as above), in whom MTB was isolated incidentally (BACTEC or culture of bronchial sample) during routine diagnostic bronchoscopy performed at the beginning of diagnostic route of sarcoidosis. Examination of bronchial washings for the presence of MTB is a routine practice in patients suspected of sarcoidosis, but in all these subjects the positive result was an unexpected finding. The follow-up observation and clinical data confirmed false negative MTB culture/ /BACTEC results. All these patients were smearnegative, and in all of them, the second sample was smear- and culture-negative. Historical TST was also negative in all individuals. All had negative history of TB and denied a contact with any subject suffering from active TB. This subgroup will be further indicated as $\mathrm{S}_{\mathrm{MTB(false+}+}$. TB in these patients was considered highly improbable, mainly due to inconsistent clinical (patients 2,4,5 with acute onset sarcoidosis), and radiological presentations (patients 1,2,4,5 with no signs of parenchymal involvement, only enlarged hilar bilateral lymph nodes). A follow-up evaluation also revealed inconsistent reaction to anti-TB treatment (progression despite treatment in patients 3 and 6 ) or to the absence of treatment (full recovery in patients 1 and 4, despite lack of anti-TB treatment). Four patients were treated with anti-TB drugs (isoniazid $5 \mathrm{mg} / \mathrm{kg}$, rifampicin $10 \mathrm{mg} / \mathrm{kg}$ (no more than $0.6 \mathrm{~g} /$ day), pyrazinamide $25 \mathrm{mg} / \mathrm{kg}$ and ethambutol $15 \mathrm{mg} / \mathrm{kg}$ ): patients 3 and 6 due to progressive parenchymal changes associated with positive culture results and the patient 5 with very low probability of TB infection but due to high potential epidemiological threat (primary school teacher), and in the patient 2 also with very low TB probability, the decision was made by a pulmonologist from another institution. In four patients, oral prednisone (initially $0.5 \mathrm{mg} / \mathrm{kg}$ ) was introduced after the diagnosis of sarcoidosis had been established (patients 1, 2, 3 and 6), but before blood sample for QFT was taken. The demographic and clinical data are provided in Table 1, and the detailed characteristics of $S_{\text {MTB(false+) }}$ patients in Table 2. 
Table 1. Characteristics of the study group consisted of sarcoidosis patients with negative culture/BACTEC results (Sarc ${ }_{\mathrm{MTB}(-)}$ group) and sarcoidosis with positive culture/BACTEC results (Sarc $_{\mathrm{MTB}(\text { false+) }}$ group)

\begin{tabular}{|c|c|c|c|c|c|}
\hline Group & $\begin{array}{c}\text { Age (years) } \\
\text { mean (SD) }\end{array}$ & Gender & $\begin{array}{c}\text { Initial } \\
\text { X-ray stage }\end{array}$ & $\begin{array}{c}\text { LS } \\
\text { at onset }\end{array}$ & $\begin{array}{c}\text { Active disease } \\
\text { at } V(0)\end{array}$ \\
\hline $\begin{array}{l}\text { Sarc }_{\text {MTB(-) }} \\
(n=37)\end{array}$ & $44(11)$ & $\begin{array}{l}F-13(35 \%) \\
M-24(65 \%)\end{array}$ & $\begin{array}{c}\text { I- } 16(43 \%) \\
\text { II }-20(54 \%) \\
\text { III }-1(3 \%) \\
\text { IV }-0(0 \%)\end{array}$ & $12(32 \%)$ & 30 (81\%) \\
\hline $\begin{array}{l}\operatorname{SarC}_{\text {MTB(false }+1} \\
(n=6)\end{array}$ & $44(4.7)$ & $\begin{array}{l}F-2(33 \%) \\
M-4(67 \%)\end{array}$ & $\begin{array}{l}I-4(67 \%) \\
\text { II }-2(33 \%)\end{array}$ & $3(50 \%)$ & $2(33 \%)$ \\
\hline
\end{tabular}

F: female; LS: Löfgren syndrome; M: male; MTB: mycobacterium tuberculosis; S: sarcoidosis; SD: standard deviation

Table 2. Detailed characteristics of sarcoidosis patients with positive culture or BACTEC results from bronchoscopy sample (Sarc $\mathrm{MTB}(+)_{\text {group) }}$

\begin{tabular}{|c|c|c|c|c|c|c|c|c|c|}
\hline Patient & Gender & Age & $\begin{array}{l}\text { X-ray stage } \\
\text { at onset }\end{array}$ & $\begin{array}{l}\text { LS at } \\
\text { onset }\end{array}$ & $\begin{array}{c}\text { Extra- } \\
\text {-pulmonary } \\
\text { sarcoidosis }\end{array}$ & $\begin{array}{l}\text { Method of } \\
\text { MTB } \\
\text { assessment }\end{array}$ & $\begin{array}{c}\text { Sarcoidosis } \\
\text { activity } \\
\text { at } V(0)\end{array}$ & $\begin{array}{c}\text { Anti-TB } \\
\text { treatment }\end{array}$ & Follow-up \\
\hline 1 & $\mathrm{M}$ & 45 & I & No & Eyes & BACTEC & Inactive & No & Recovery \\
\hline 2 & $\mathrm{M}$ & 37 & I & Yes & No & BACTEC & Inactive & Yes & Recovery \\
\hline 3 & $\mathrm{~F}$ & 41 & II & No & No & $\begin{array}{c}\text { Culture } \\
3 \text { colonies }\end{array}$ & Active & Yes & $\begin{array}{c}\text { Chronic pro } \\
\text { gressive }\end{array}$ \\
\hline 4 & $\mathrm{M}$ & 47 & I & Yes & No & BACTEC & Inactive & No & Recovery \\
\hline 5 & $\mathrm{~F}$ & 48 & I & Yes & No & $\begin{array}{l}\text { Culture } \\
2 \text { colonies }\end{array}$ & Inactive & Yes & Recovery \\
\hline 6 & $\mathrm{M}$ & 47 & II & No & No & $\begin{array}{c}\text { Culture } \\
2 \text { colonies }\end{array}$ & Active & Yes & $\begin{array}{l}\text { Chronic } \\
\text { progressive }\end{array}$ \\
\hline
\end{tabular}

In patients 2, 3, 5, 6 the decision of anti-TB treatment was made in a consequence of the positive culture or BACTEC result obtained during diagnostic procedures of suspected sarcoidosis. Radiological changes recovered completely in patients 1 and 4 without anti-TB treatment. In patients 3 and 6 radiological changes progressed despite anti TB-treatment; F: female; M: male; TB: tuberculosis

The control group

The control group ( $\mathrm{n}=37,17$ females $)-$ consisted of inhabitants of Lodz city and Lodz province. The group was composed of healthy volunteers, age-matched to the sarcoidosis group. All subjects completed the questionnaire concerning individual TB history, and the answers to all questions were negative. In all patients, chest X-ray was normal, or in case of abnormal chest $\mathrm{x}$-ray, tuberculosis could be unambiguously excluded. None of these patients were treated with systemic steroids or immunosuppressive drugs, nor suffered any disease connected with depleted immune response. None of the subjects had a positive history of sarcoidosis.

The TB low- risk group

Medical students ( $\mathrm{n}=21,6$ females) with no history of TB or TB contacts, as assessed by a TB questionnaire. Chest X-ray taken during the last 12 months was normal.

The active TB group

Patients ( $\mathrm{n}=23,9$ females) actually treated due to lung tuberculosis (between $2^{\text {nd }}$ and $6^{\text {th }}$ week of treatment), with typical clinical and radiological picture and positive sputum or bronchial washing culture results.

Sarcoidosis patients were followed up for at least 2 years (regular every 3-6-month visits in out-patient department and telephone check-ups).

All subjects participating in the study were provided with relevant information, after which they signed the consent. The Ethical Committee at Medical University of Lodz approved the protocol (RNN/110/14/KE).

QuantiFERON-TB GOLD In-tube (QFT) was performed according to the manufacturer's in- 
Table 3. Results of QuantiFERON TB GOLD test in all subgroups — Fisher's exact test

\begin{tabular}{lcccccc}
\hline \multicolumn{1}{c}{ Group } & Positive & Negative & All & $\%$ & $\begin{array}{c}\text { Versus control group } \\
\text { (p-value) }\end{array}$ & $\begin{array}{c}\text { Versus low TB risk group } \\
\text { (p-value) }\end{array}$ \\
\hline Sarc $_{\text {MTB(false+) }}$ & 0 & 6 & 6 & 0 & 1.0 & 1.0 \\
Sarc $_{\text {MTB-) }}$ & 9 & 28 & 37 & 24.3 & 0.37 & 0.08 \\
Sarc (entire) & 9 & 34 & 43 & 20.9 & 0.56 & 0.15 \\
Control & 5 & 32 & 37 & 13.5 & - & 0.4 \\
TB active & 16 & 7 & 23 & 69.6 & - & -0.00001 \\
Low TB risk & 1 & 20 & 21 & 4.8 & - & - \\
\hline
\end{tabular}

struction (QIAGEN, Germany). Briefly, $5 \mathrm{ml}$ of blood was withdrawn from a cubital vein into three collecting tubes (Nil tube, MTB tube, mitogen tube). Samples were incubated and centrifuged. Afterwards plasma was collected and frozen in $-20^{\circ} \mathrm{C}$ until measurements. After defrosting, the samples were transferred onto 96-wells plate and tested for the presence of interferon gamma (IFN- $\gamma$ ) by ELISA method. Optical density (OD) of each well was measured using multiplate reader BioTek ELx 800 equipped with a $450-\mathrm{nm}$ filter $(620 \mathrm{~nm}$ for reference). OD values were taken to calculate the results, according to standard curve drawn basing on provided standards. The results were interpreted as positive, negative or indeterminate.

Frequencies of positive and negative outcomes were calculated for sarcoidosis with negative TB results, positive TB results, and for entire sarcoidosis group. These values were compared with those of dedicated control group and of low-TB risk group, using the Fisher's exact test.

\section{Results}

The incidence of positive QFT results among sarcoidosis patients with negative culture/BACTEC results for MTB ( Sarc $_{\mathrm{MTB}(-)}$ ) was slightly higher than in the control group of age-matched non-sarcoid subjects, however, the difference did not reach the level of statistical significance $(p=0.37)$. None of the sarcoidosis patients with positive culture/BACTEC results for MTB ( SarC $_{\text {MTB(false+t) }}$ ) had positive QFT result. When results of the entire sarcoidosis group $\left(\operatorname{SarC}_{\mathrm{MTB}(-)}\right.$ and $\operatorname{SarC}_{\mathrm{MTB}(\text { false++)})}$ were calculated, still no differences compared to controls were found. In fact, the level of significance was even lower, due to the decrease of the percentage of positive results in the entire sarcoidosis group $(\mathrm{p}=0.56)$. Table 3 shows detailed results within all study groups.

\section{Discussion}

In our outcomes, the frequency of latent tuberculosis infection based on QFT results is not significantly higher when compared to age-matched controls. Results obtained by other authors are similar: low incidence of positive results among Danish sarcoidosis patients [11] or high (however, corresponding to high incidence of TB in the studied society) frequency of positive results documented by authors from India [12]. In the study performed by other researchers among Polish, formerly vaccinated sarcoidosis patients, the percentage of positive results was 4.6 when assessed by QFT, and 3.7 when assessed by T-SPOT TB test [13]. Although the percentage of positive outcomes in our sarcoidosis group was slightly higher than in the control group, it well matched the $20.4 \%$ prevalence of latent TB reported for Polish blood donors [15]. Therefore, it is justified to say that the frequency of latent TB infection in sarcoidosis patients reflects the one reported in the general population, from which sarcoidosis patients originate.

By using QFT we were able to show negative QFT results in all sarcoidosis patients with highly suspected false positive culture results. Tuberculosis in these patients was recognized as unlikely using similar approach as Fischl et al. [16], therefore using clinical outcome, despite an initial positive culture for M. tuberculosis. Although such approach would be recognized as controversial, careful follow-up justified rightness of our decision. We emphasize that QFT negative result alone cannot be used as a proof of falsenegative MTB culture, however, in confrontation with the overall clinical description, it was recognized as potentially useful additional diagnostic information. To the best of our knowledge, this is the first study with the use of QFT in sarcoidosis patients, which also included those with positive culture results. 
Among the sarcoidosis group with positive culture results, in all patients, the possibility of TB infection was considered as very low. Therefore, in two of them, the decision to abandon the anti-TB treatment was taken. Both patients with stage I disease were free of symptoms and their chest $\mathrm{X}$-rays were normal at follow-up examination, even though one was treated with systemic steroids for over 12 months due to ocular sarcoidosis. In the remaining two patients with stage I sarcoidosis, the anti-TB treatment was introduced (Pt 5, a primary school teacher - due to potential high epidemiologic threat, and Pt 2, following decision of a pulmonologist representing another center, and his opinion on this matter was not shared by our group). Systemic steroids were applied in 4 patients. In none systemic steroids were introduced prior to obtaining the positive culture/BACTEC results.

As the interpretation, two possibilities should be considered: the occurrence of false negative results of QFT or false positive results of bronchial aspirate culture. The pooled analysis of QFT results from different studies revealed the sensitivity of $80 \%$ and specificity of $79 \%$ [17]. Also, in other studies, false negative IGRA results are not rare in active TB $[18,19]$. At risk are small children with severe TB forms, those with reduced number and function of lymphocytes [20-22], patients in immunosuppression related to different diseases, lower platelet, protein and albumin levels, or senility [19-22]. None of the study subjects presented any of the described conditions. Therefore, false-negative QFT results may not be excluded with full confidence. A second possibility are false positive sputum-culture results for MTB. It is not surprising, as specimen cross-contamination with MTB was reported in many laboratories [6, 23, 24]. A cross-contamination of TB strains was studied in our city by the use of genotyping methods, and was found in 4 out of 98 clustered strains [25]. Taking into consideration all clinical, radiological, and laboratory results, we are convinced of false culture results in all these sarcoidosis patients. The most important features in support of this interpretation and present in our patients in variable constellations, are: predominance of intrathoracic lymph nodes involvement over parenchymal disease (isolated lymphonodal tuberculosis may happen in primary TB only, therefore it is unlikely in adult members of TB vaccinated society), lack of endobronchial changes suggestive of TB, acute onset, complete resolution of radiological changes despite lack of anti-TB treatment, and radiological progression regardless of anti-TB treatment.
Our observations strengthen the suggestion that clinicians should not alwaysconfidently repeat clichés, but rather evaluate positive cultures critically, because such approach would allow to decrease the probability of unnecessary treatment and its possible complications.

Sarcoidosis is characterized by enhanced local immunological reactions and consequent peripheral anergy (immunological paradox) [26]. $\mathrm{T}$ regulatory cells (CD4+CD25+FOXP3 + and CD4+CD39+) may be involved, as they incompletely control the inflammation of injured tissues, but are powerful enough to mediate peripheral anergy [27, 28]. IGRAs performed on whole blood (QFT) or peripheral blood mononuclear cells (T-SPOT TB) could theoretically be negative due to activity of these cells but interestingly, it was shown that nTreg cells incompletely blocked IFN-gamma and TNF secretion by autologous cells [27]. As several patients of our group with active disease were QFT-positive, the possibility of false negative results due to this phenomenon is unlikely. It is worth noticing that the new version of QFN - QuantiFERON TB Plus accessing in addition to CD4+ T-cell response also CD8+ T-cell response, which can better discriminate active TB from LTBI [29, 30], as well as recent exposure to TB [31] will be especially useful in situation like ours.

One limitation of our study was a small number of sarcoidosis patients with false-positive culture results. Another weakness was lack of sarcoidosis patients with true concurrent TB, which would allow to verify the sensitivity and specificity of QFT in this specific clinical situation. However, such patients are rare, and it seems improbable to recruit satisfactory sample of such subjects.

\section{Conclusion}

QFT has been found to be the useful test for the detection of latent TB infection in sarcoidosis patients. In addition, we confirm that sarcoidosis activity does not negatively influence the result of QFT. Moreover, QFT would be proposed as a cost-saving diagnostic test providing additional diagnostic information when false positive MTB culture result in the sarcoidosis patient is highly suspected. However, in each case clinical, radiological and epidemiological data should be considered before taking the therapeutic decision. 


\section{Conflict of interest}

\section{The authors declare no conflict of interest.}

\section{References:}

1. Dubaniewicz A. Microbial and human heat shock proteins as ,danger signals' in sarcoidosis. Hum Immunol. 2013; 74(12): 1550-1558, doi: 10.1016/j.humimm.2013.08.275, indexed in Pubmed: 23993988.

2. Piotrowski WJ, Górski P, Duda-Szymańska J, et al. Mycobacterium tuberculosis as a sarcoid factor? A case report of family sarcoidosis. Am J Case Rep. 2014; 15: 216-220, doi: 10.12659/ AJCR.890014, indexed in Pubmed: 24847413.

3. Koth LL, Solberg OD, Peng JC, et al. Sarcoidosis blood transcriptome reflects lung inflammation and overlaps with tuberculosis. Am J Respir Crit Care Med. 2011; 184(10): 1153-1163, doi: 10.1164/rccm.201106-1143OC, indexed in Pubmed: 21852540.

4. Maertzdorf J, Weiner J, Mollenkopf HJ, et al. TBornotTB Network. Common patterns and disease-related signatures in tuberculosis and sarcoidosis. Proc Natl Acad Sci U S A. 2012; 109(20): 7853-7858, doi: 10.1073/pnas.1121072109, indexed in Pubmed: 22547807.

5. Agrawal R, Kee AeRa, Ang L, et al. Tuberculosis or sarcoidosis: Opposite ends of the same disease spectrum? Tuberc Edinb Scotl. 2016; 98: 21-26.

6. Lee MR, Chung KP, Chen WT, et al. Epidemiologic surveillance to detect false-positive Mycobacterium tuberculosis cultures. Diagn Microbiol Infect Dis. 2012; 73(4): 343-349, doi: 10.1016/j.diagmicrobio.2012.04.014, indexed in Pubmed: 22705229.

7. Smith-Rohrberg D, Sharma SK. Tuberculin skin test among pulmonary sarcoidosis patients with and without tuberculosis: its utility for the screening of the two conditions in tuberculosis-endemic regions. Sarcoidosis Vasc Diffuse Lung Dis. 2006; 23(2): 130-134, indexed in Pubmed: 17937109.

8. Diel R, Goletti D, Ferrara G, et al. Interferon- $\gamma$ release assays for the diagnosis of latent Mycobacterium tuberculosis infection: a systematic review and meta-analysis. Eur Respir J. 2011; 37(1): 88-99, doi: 10.1183/09031936.00115110, indexed in Pubmed: 21030451.

9. Goletti D, Carrara S, Stefania C, et al. Accuracy of immunodiagnostic tests for active tuberculosis using single and combined results: a multicenter TBNET-Study. PLoS One. 2008; 3(10): e3417, doi: 10.1371/journal.pone.0003417, indexed in Pubmed: 18923709.

10. Goletti D, Carrara S, Vincenti D, et al. Accuracy of an immune diagnostic assay based on RD1 selected epitopes for active tuberculosis in a clinical setting: a pilot study. Clin Microbiol Infect. 2006; 12(6): 544-550, doi: 10.1111/j.1469-0691.2006.01391.x, indexed in Pubmed: 16700703.

11. Milman N, Søborg B, Svendsen CBo, et al. Quantiferon test for tuberculosis screening in sarcoidosis patients. Scand J Infect Dis. 2011; 43(9): 728-735, doi: 10.3109/00365548.2011.582141, indexed in Pubmed: 21619424.

12. Gupta D, Kumar S, Aggarwal AN, et al. Interferon gamma release assay (QuantiFERON-TB Gold In Tube) in patients of sarcoidosis from a population with high prevalence of tuberculosis infection. Sarcoidosis Vasc Diffuse Lung Dis. 2011; 28(2): 95-101, indexed in Pubmed: 22117500.

13. Kempisty A, Białas-Chromiec B, Borkowska D, et al. Interferon gamma release assays based on $\mathrm{M}$. tuberculosis-specific antigens in sarcoidosis patients. Pneumonol Alergol Pol. 2015; 83(2): 126134, doi: 10.5603/PiAP.2015.0020, indexed in Pubmed: 25754054.

14. Statement on sarcoidosis. Joint Statement of the American Thoracic Society (ATS), the European Respiratory Society (ERS) and the World Association of Sarcoidosis and Other Granulomatous Disorders (WASOG) adopted by the ATS Board of Directors and by the ERS Executive Committee, February 1999. Am J Respir Crit Care Med. 1999; 160(2): 736-755, doi: 10.1164/ajrccm.160.2.ats4-99, indexed in Pubmed: 10430755.

15. Kuś J, Demkow U, Lewandowska K, et al. [Prevalence of latent infection with Mycobacterium tuberculosis in Mazovia Region using interferon gamma release assay after stimulation with specific antigens ESAT-6 and CFP-10]. Pneumonol Alergol Pol. 2011; 79(6): 407-418, indexed in Pubmed: 22028119.

16. Fischl MA, Uttamchandani RB, Daikos GL, et al. An outbreak of tuberculosis caused by multiple-drug-resistant tubercle bacilli among patients with HIV infection. Ann Intern Med. 1992; 117(3): 177-183, indexed in Pubmed: 1616211.

17. Sester M, Sotgiu G, Lange C, et al. Interferon- $\gamma$ release assays for the diagnosis of active tuberculosis: a systematic review and meta-analysis. Eur Respir J. 2011; 37(1): 100-111, doi: 10.1183/09031936.00114810, indexed in Pubmed: 20847080

18. Stephan C, Wolf T, Goetsch U, et al. Comparing QuantiFERON -tuberculosis gold, T-SPOT tuberculosis and tuberculin skin test in HIV-infected individuals from a low prevalence tuberculosis country. AIDS. 2008; 22(18): 2471-2479, doi: 10.1097/ QAD.0b013e3283188415, indexed in Pubmed: 19005270.

19. Kim EY, Park MS, Kim YS, et al. Risk factors for false-negative results of QuantiFERON-TB Gold In-Tube assay in non-HIV-infected patients with culture-confirmed tuberculosis. Diagn Microbiol Infect Dis. 2011; 70(3): 324-329, doi: 10.1016/j.diagmicrobio.2011.02.011, indexed in Pubmed: 21546200.

20. Altet-Gómez N, De Souza-Galvao M, Latorre I, et al. Diagnosing TB infection in children: analysis of discordances using in vitro tests and the tuberculin skin test. Eur Respir J. 2011; 37(5): 1166-1174, doi: 10.1183/09031936.00022710, indexed in Pubmed: 20729220.

21. Leidl L, Mayanja-Kizza H, Sotgiu G, et al. Relationship of immunodiagnostic assays for tuberculosis and numbers of circulating CD4+ T-cells in HIV infection. Eur Respir J. 2010; 35(3): 619-626, doi: 10.1183/09031936.00045509, indexed in Pubmed: 19608590.

22. Cho K, Cho E, Kwon S, et al. Factors Associated with Indeterminate and False Negative Results of QuantiFERON-TB Gold In-Tube Test in Active Tuberculosis. Tuberc Respir Dis (Seoul). 2012; 72(5): 416-425, doi: 10.4046/trd.2012.72.5.416, indexed in Pubmed: 23101006.

23. Bhattacharya M, Dietrich S, Mosher L, et al. Cross-contamination of specimens with Mycobacterium tuberculosis: clinical significance, causes, and prevention. Am J Clin Pathol. 1998; 109(3): 324-330, indexed in Pubmed: 9495206.

24. Lai CC, Tan CK, Lin SH, et al. Molecular evidence of false-positive cultures for Mycobacterium tuberculosis in a Taiwanese hospital with a high incidence of TB. Chest. 2010; 137(5): 1065-1070, doi: 10.1378/chest.09-1878, indexed in Pubmed: 19965955.

25. Krawczyk M, Brzostek A, Gorna A, et al. Epidemiological analysis of Mycobacterium tuberculosis strains isolated in Lodz, Poland. Int J Tuberc Lung Dis. 2011; 15(9): 1252-1258, doi: 10.5588/ ijtld.10.0718, indexed in Pubmed: 21943854.

26. Miyara M, Amoura Z, Parizot C, et al. The immune paradox of sarcoidosis and regulatory T cells. J Exp Med. 2006; 203(2): 359-370, doi: 10.1084/jem.20050648, indexed in Pubmed: 16432251.

27. Idali F, Wahlström J, Müller-Suur C, et al. Analysis of regulatory $\mathrm{T}$ cell associated forkhead box P3 expression in the lungs of patients with sarcoidosis. Clin Exp Immunol. 2008; 152(1): 127-137, doi: 10.1111/j.1365-2249.2008.03609.x, indexed in Pubmed: 18279440.

28. Chen X, Zhou B, Li M, et al. CD4(+)CD25(+)FoxP3(+) regulatory T cells suppress Mycobacterium tuberculosis immunity in patients with active disease. Clin Immunol. 2007; 123(1): 50-59, doi: 10.1016/j.clim.2006.11.009, indexed in Pubmed: 17234458.

29. Rozot V, Patrizia A, Vigano S, et al. Combined use of Mycobacterium tuberculosis-specific CD4 and CD8 T-cell responses is a powerful diagnostic tool of active tuberculosis. Clin Infect Dis. 2015; 60(3): 432-437, doi: 10.1093/cid/ciu795, indexed in Pubmed: 25362202.

30. Day CL, Abrahams DA, Lerumo L, et al. Functional capacity of Mycobacterium tuberculosis-specific T cell responses in humans is associated with mycobacterial load. J Immunol. 2011; 187(5): 2222-2232, doi: 10.4049/jimmunol.1101122, indexed in Pubmed: 21775682 .

31. Barcellini L, Borroni E, Brown J, et al. First evaluation of QuantiFERON-TB Gold Plus performance in contact screening. Eur Respir J. 2016; 48(5): 1411-1419, doi: 10.1183/13993003.005102016, indexed in Pubmed: 27390280. 